\title{
THE DEVELOPMENT OF S-BAND PLANE WAVE TRANSFORMER PHOTOINJECTOR *
}

\author{
X. Ding, C. Pellegrini, J. Rosenzweig, S. Telfer, A. Tremaine, W. Vernon \\ Department of Physics and Astronomy, UCLA, LA, CA90095-1547 \\ D. Yu, D. Newsham, J. Zeng, T. Lee, J. Chen \\ DULY Research Inc.
}

\begin{abstract}
An integrated S-Band RF photoinjector based on the plane wave transformer (PWT) is being built in the Particle Beam Physics Laboratory at UCLA in collaboration with DULY Research. This novel structure integrates a photocathode directly into a PWT linac [1] making the structure simple and compact. Due to the strong coupling between each adjacent cell, this structure is relatively easy to fabricate and operate [2]. This photoinjector can provide high brightness beams at energies of 15 to $20 \mathrm{MeV}$, with emittance less than $1 \mathrm{~mm}$ mrad at charge of $1 \mathrm{nC}$ [3]. These short-pulse beams can be used in various applications: space charge dominated beam physics studies, plasma lenses, plasma accelerators, free-electron laser microbunching techniques, and SASEFEL physics studies [4]. It will also provide commercial opportunities in chemistry, biology and medicine. The present status of the PWT photoinjector including fabrication and cold test to characterise the structure is described. RF system and photocathode drive laser system are also discussed.
\end{abstract}

\section{INTRODUCTION}

The photoinjector concept was first proposed and experimentally tested at Los Alamos in mid-80s. Photoinjectors have proven their ability to produce very bright electron beams. The high brightness beams have several applications, such as self-amplified spontaneous emission free-electron lasers (SASE-FEL), Compton scattering sources, wake-field accelerator drivers, and linear collider sources of polarized electrons.

The conventional BNL/SLAC/UCLA 1.625 cell photocathode RF gun has been proved to be a reliable high brightness beam source [5], but the beam energy of this type of gun is relative low $(4 \sim 5 \mathrm{MeV})$, it usually needs additional accelerating sections to get relative high energy. And due to its small cell-to-cell coupling and small mode separation $(3 \mathrm{MHz})$, it is hard to make.

A new S-band photoinjector design is pursued by a UCLA/DULY Research collaboration. A novel compact standing wave RF structure (PWT) is integrated with a removable photocathode. This structure has great coupling between the accelerating cells. The mechanical tolerances for the PWT gun is much higher than those for the BNL/SLAC/UCLA type gun, making it much easier to fabricate.

The S-band PWT photoinjector is a Small Business Innovation Research (SBIR) project proposed by DULY research Inc. teaming up with UCLA to develop a novel photoelectron linear accelerator. The goal of this project is to accelerate a short pulse $(\sim 5 \mathrm{ps})$, low emittance $(\sim 1 \mathrm{~mm}-\mathrm{mrad})$, high charge $(1 \mathrm{nC})$ electron beam in a compact $(\sim 33 \mathrm{MeV} / \mathrm{m})$, low $\sim$ cost linear accelerator. The compact linac will have broad commercial and research applications.

A schematic of the PWT photoinjector is shown in Fig.1. The structure consists of a cylindrical tank and an array of disks. There are totally 10 full cells and 2 half cells in the whole structure. The disks are connected together by 4 metal rods parallel to the axis. The disks are separated from the cylindrical tank, so they are like a centre conductor to support a TEM-like plane wave between the tank and disks. The field within the region of the disk is similar to a TM01 mode, which can accelerate electrons on the axis. This structure transforms a transverse plane wave field into a field having a longitudinal electric component for acceleration of electrons. The feature causes the PWT structure to have the advantages of high shunt impedance and strong coupling between each cell. The PWT also provides good vacuum conductance and easy fabrication. But this structure also raises the concern about the field asymmetry caused by the four supporting/cooling rods. Our simulation and cold test shows this effect is negligible.

In this paper, we describe the RF structure design, focusing solenoid design, beam dynamics study, RF system, laser system and current status of the PWT gun.

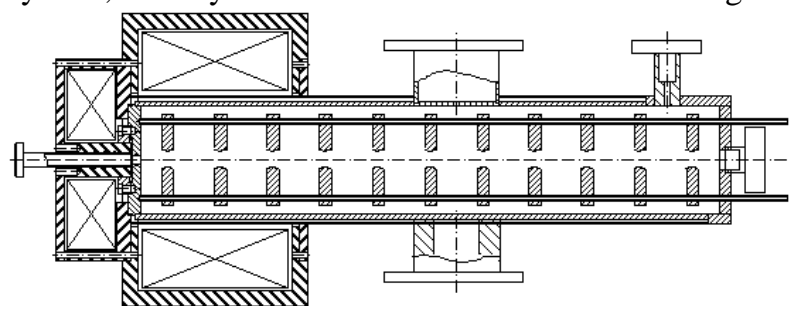

Figure 1: Schematic view of the PWT photoinjector.

"Work supported by U.S. DOE grants DE-FG03-98ER45693 and SBIR DE-FG03-96ER82156. 


\section{ELECTROMAGNETIC STRUCTURE DESIGN}

The PWT structure is simulated by using both SUPERFISH and GDFIDL. The SUPERFISH code is a 2D electromagnetic field solver; it is helpful in finding dimensions of PWT for a given frequency. However, the metal rods cannot be included because the code is lack of ability to solve 3D problem. Therefore, the 3D code GDFIDL has been used to obtain a more complete picture of the RF field and it gives a more precise value of the properties of the RF structure.

Figure 2(a) shows a field plot from SUPERFISH and GDFIDL, a clear plane wave (TEM-like) pattern is shown between the outer tank and the disk assembly. The acceleration electric field distribution (TM01-like) is shown at the centre of the disk irises. Figure 2(b) shows the feature of electric field contours on the transverse cross-section at the centre of the full cell. It should be noted that the rods (not shown on Figure) only produce a minor on-axis perturbation on the field distribution for this operation mode. Especially for the field closed to the beam centre, this perturbation is negligible. A detailed study of this perturbation is shown in another conference paper by J.Rosenzweig et al [6].

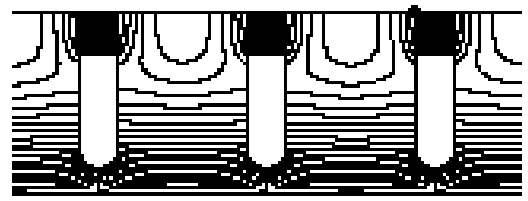

(a)

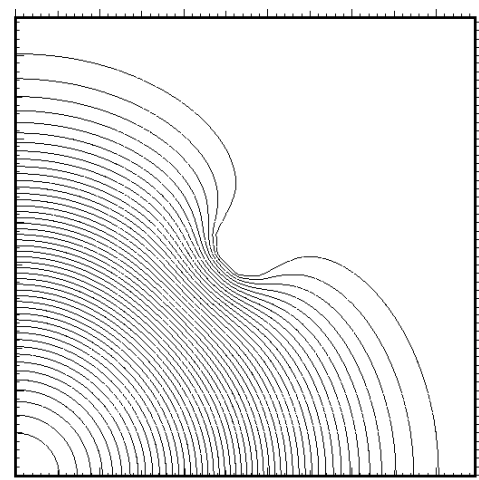

(b)

Figure 2: (a) Plots of the Electric field distribution of the operating mode at the on-axis cross-sections. (b) The feature of axial direction electric field contour.

In the beam dynamics simulation, we first make an electric field map from SUPERFSH and GDFIDL and a magnetic field map from POISSON. Then we run PARMELA to get a good electron beam simulation. This design is subject to the following constraints:
- Relatively high shunt impedance;

- Minimizing higher spatial harmonic content;

- Cell number and gradient appropriate to deliver $20 \mathrm{MeV}$ beam;

- Good coupling and high mode separation;

- Low transverse multipole content;

- Relatively low Q to allow for structure filling;

- Relatively small outer diameter to allow compact focusing solenoid;

Given all of there constraints, we have chosen an inner radius of the tank wall to be $5.5 \mathrm{~cm}$ Disks have donuts shapes with ID irises of $1.6 \mathrm{~cm}$, which can minimize higher spatial harmonic content and provide $55 \mathrm{M} \Omega / \mathrm{m}$ shunt impedance. According to a recent analytical theory of emittance compensation, the solenoid magnetic field should start focusing as early as possible, and the emittance compensation prefers a relatively low field gradient. In our case we choose the on-axis maximum field to be $60 \mathrm{MV} / \mathrm{m}$. With a $24 \mathrm{MW}$ input power, We decide to use $10+2 / 2$ cells $(57.75 \mathrm{~cm})$ structure to obtain $20 \mathrm{MeV}$ electrons. The coupling coefficient is mainly dictated by the distance from the outer radius of the disks and the inner radius of the tank. For the parameters we have chosen, the coupling was quite strong, with 0 and $\pi$ mode separation about $400 \mathrm{MHz}, 10 / 11 \pi$ and $\pi$ mode over $8 \mathrm{MHz}$. The quality factor $\mathrm{Q}$ is around 20,000 . That allows for the structure filling time less than $4 \mu \mathrm{s}$. A solenoid field map is shown in Figure 3.

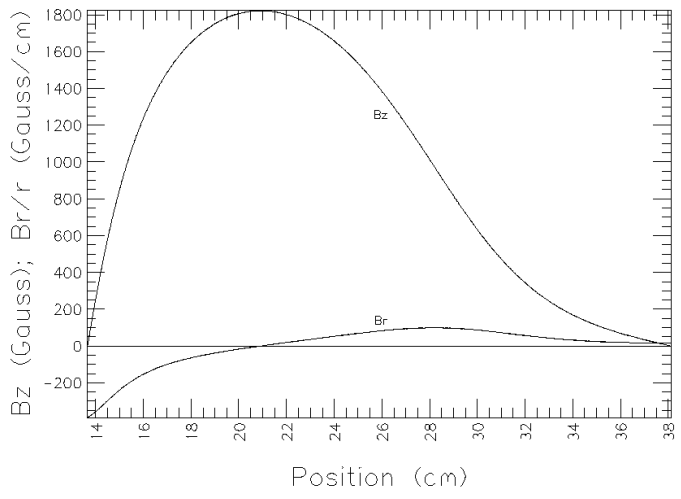

Figure 3: Magnetic field distribution along the axis.

\section{BEAM DYNAMIC STUDY}

The beam optics design is closely related to the RF structure design and the focusing solenoid design. The purpose of the beam dynamics design is for obtaining the highest brightness beam with minimal emittance. The basic technique for the beam dynamics design is experimenting with different electron input and electromagnetic fields layout, using code such as PARMELA to get the best beam output. Based on the Serafini and Rosenzweig (SR) theory, we can get good 
emittance compensation if the beam profile follows the invariant envelope, which has the form:

$$
\sigma=\frac{2 m_{e} c^{2}}{E} \sqrt{\frac{I}{3 I_{0} \gamma(z)}}
$$

where $\mathrm{E}$ is the average acceleration field, $\mathrm{I}$ is the rms current, and $\mathrm{I}_{0}=17 \mathrm{kA}$. The theory prefers a low gradient $(60 \mathrm{MV} / \mathrm{m})$ and a nominal launch phase $\phi_{0}=32^{\circ}$ to match the invariant envelope. Besides considering this matching, we also have other constraints in our beam dynamic design: beam charge $1 \mathrm{nC}$ to be ideal for FEL experiment, frequency $2856 \mathrm{MHz}$ to match the RF power provided by S-Band klystron, pulse length 9.8psec FWHM by availability of the laser source. Figure 4 displays the evolution of the rms beam size and emittance from the PARMELA simulation and invariant envelope equations.

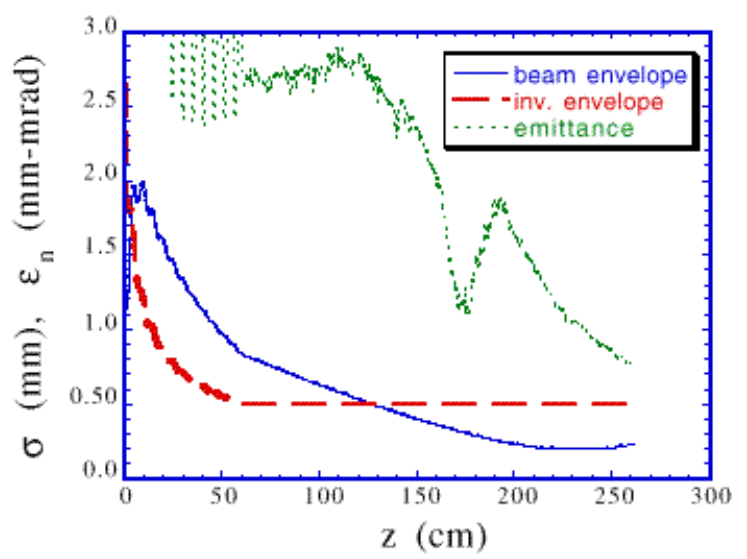

Figure 4: Comparison of beam envelope, emittance from PARMELA and invariant envelope from equation (1).

According to a scaling theory of RF gun design, this injector can also run at low charge $(15 \mathrm{pC})$, ultra-low emittance $(0.06 \mathrm{~mm}-\mathrm{mrad})$ mode, which can be used as an injector for the plasma beatwave acceleration and optical acceleration experiments.

\section{THE COLD TEST RESULTS}

To test what we found in the numerical simulation, a cold test piece that characterises the PWT structure was made. The test piece consists of 2 half cells shown in Figure 5. All measurements were done with a HP network analyser. The on-axis electric field distribution is measured by a bead-pull perturbation technique. To reduce the system error in the measurements, the room temperature has to be regulated with very small fluctuation. The $\pi$ mode frequency from the cold test is $2856.556 \mathrm{MHz}$. The GDFIDL simulation gives us $2855.820 \mathrm{MHz}$ for the chosen dimensions. The difference is less than $0.03 \%$. We also measured the mode separation between the $\pi$ mode and 0 mode which is over $400 \mathrm{MHz}$. The real PWT structure has been machined and copper plated on the tank inner surface and metal rods. The whole structure (10 full cells and 2 half cells)will be brazed together and be tested soon.

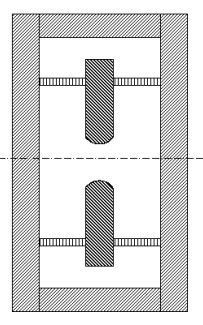

(a)

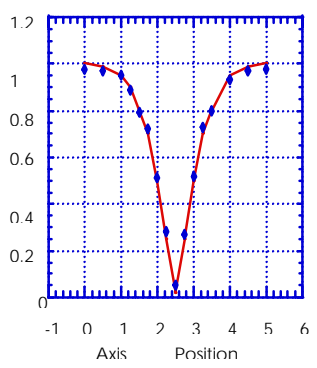

(b)
Figure 5: (a)Schematic of test piece (b) Electric field distrubution on axis, line value from simulation, dot value from bead pull measurement.

\section{SUPPORT SYSTEMS}

\subsection{RF Systems}

The RF system supplies the power to drive the PWT gun. A $2856 \mathrm{MHz}$ low-level signal $(\sim \mathrm{mW})$ is generated by the laser/timing system and amplified by a solid state amplifier to 300W. A SLAC XK5 type klystron driven by a modulator uses this signal to produce up to $24 \mathrm{MW}$ RF powers in a $4 \mu$ s pulse. The output power is controlled by adjusting the high voltage supplied to the modulator. A high power isolator and a RF window are installed between the gun and the klystron to protect the klystron.

\subsection{Laser System}

A new Lightwave 131 laser with all-diode pumped $\mathrm{Nd}$ :YLF regenerative amplifier was developed and installed at UCLA, in collaboration with Lightwave electronics. This system provides 10ps FWHM pulses without CPA. Doubling crystals are available to provide UV conversion. We can get $300 \mu \mathrm{J}$ UV (wavelength $263 \mathrm{~nm})$ pulse, more than enough energy to make the PWT gun to yield over $1 \mathrm{nC}$ of charge from a copper photocathode.

\section{REFERENCES}

[1] R. Zhang, R. Cooper and C. Pellegrini, "Study of a Novel Compact Standing Wave RF Linac", in course of publications in Nuclear Instruments and Methods in Physics Research, Section A (1996)

[2] D.Yu, et.al. Proc. of the 1997 particle accelerator conf., 2802 (1997)

[3] J. Rosenzweig, et.al. Proc. of the 1997 particle accelerator conf.,1968(1997)

[4] M.Hogan, et.al, Phys. Rev. Lett. 80,289(1998)

[5]D.T.Palmer, et al., Proc.1997 Part. Accel. Conf. 2687(1998)

[6]J.Rosenzweig, et al. Field Asymmetry Study, this proceeding 